\title{
La metáfora de la corporalidad en la guerra como dispositivo
} resiliente*

The Metaphor of Corporality in War as a Devise of Resilience

\author{
Angélica María Villamil Benavides* \\ Universidad de Cundinamarca, Colombia \\ https://orcid.org/0000-0003-2548-5342 \\ Adrián David Galindo Ubaque \\ Universidad de Cundinamarca, Colombia \\ https://orcid.org/0000-0002-1125-9400
}

Artículo de investigación

Fecha de recepción: 15 de julio de 2019 Fecha de aceptación: 17 de octubre de 2019

\section{Para citar este artículo}

Villamil Benavides, A. M. y Galindo Ubaque, A. D. (2020). La metáfora de la corporalidad en la guerra como dispositivo resiliente. Campos en Ciencias Sociales, 8(1), 85-114. DoI: https://doi.org/10.15332/25006681/5717

* Este artículo es producto de la investigación-intervención llevada a cabo como opción de grado bajo la modalidad de monografía para optar por el título académico del programa de Psicología de la Universidad de Cundinamarca, realizada por Angélica María Villamil Benavides y asesorada por el docente Adrián David Galindo Ubaque.

** Psicóloga. Correo electrónico: amvillamil@ucundinamarca.edu.co

*** Psicólogo y magíster en Psicología Clínica y de la Familia. Correo electrónico: adavidgalindo@ucundinamarca. edu.co 


\section{REsUMeN}

Con la presente investigación-intervención se busca observar el conflicto armado colombiano desde la interpretación del excombatiente en su componente psicológico. En este sentido, se propone comprender la construcción narrativa de la resiliencia en relación con la corporalidad e identidad en un excombatiente de la guerrilla de las Farc-EP desde el marco del conflicto armado en Colombia, reconociendo la militancia del participante. A nivel metodológico se llevaron a cabo escenarios conversacionales y sensoriales que organizaron la narrativa como herramienta vital, de manera que se configuró y dinamizó el proceso y la transición en el contexto investigativo e interventivo. Este último contexto favoreció el proceso de resiliencia a través del relato emergente; luego, se retroalimentó con el modelo teórico para crear escenarios y estrategias que facilitaron el desarrollo del encuentro. Como resultado, se construyeron metáforas y reflexiones sobre la experiencia vital, el sentido del equilibrio como figura de la salud mental, el relato de la herida de guerra que enmarca la corporalidad en el proceso resiliente y el vínculo familiar con el grupo armado.

Palabras clave: corporalidad, excombatiente, identidad, narrativa, posconflicto, resiliencia.

\section{Abstract}

This research/intervention aims at observing the Colombian armed conflict from perspective of the psychological component of the ex-combatant's interpretation. In this sense, it propounds to understand the narrative construction of resilience in relation to corporality and identity of an ex-combatant of the Farc-ep guerrilla, within the framework of the armed conflict in Colombia and taking into account the participant's militancy. Methodologically, conversational and sensory scenarios were carried out, scenarios meant to organize the narrative as a vital tool, so as to inform and dynamize the process and the transition in the research and intervention context. The latter is the methodological core of research, since intervention fosters the resilience process through the emerging story; subsequently, the research/intervention methodological resource was enriched with feedback form the theoretical modelling in order to create scenarios and strategies to facilitate the development of the encounter. The most representative results of the research were the construction of metaphors and reflections on the vital experience, the sense of balance as a figure of mental 
health, the story of the war wound as framed by corporality in the resilient process, and the family bond with the armed group.

Keywords: corporality, ex-combatant, identity, narrative, post-conflict, resilience.

\section{INTRODUCCIÓN}

La investigación que se presenta en este artículo indaga por el factor psicológico resiliente y, en torno a este, la corporalidad e identidad como dispositivo narrativo en la población excombatiente de las Farc-EP. En el marco del conflicto armado, se identifica la relevancia de que, desde la academia, se amplíe el foco visual, y se extienda la investigación a voces y relatos que hasta el momento han sido desconocidos por su papel activo como actores del conflicto. Al respecto, la consolidación de los escenarios permite procesos de construcción de paz y propician encuentros con la reconciliación nacional.

Aristizábal (2015) evidencia que la población excombatiente también es sujeto de afectación psicológica tras su paso por el grupo armado y su eventual participación bélica. Esto significa que los excombatientes también pueden desarrollar traumas propios de las dinámicas de los enfrentamientos y las actividades relacionadas a dicho contexto, como sucede con los veteranos de guerra y la población víctima a raíz de las situaciones de conflicto.

Son pocos los estudios que, como el de Aristizábal, reflejan una problematización psicológica del excombatiente, elemento que se hace urgente en la construcción de paz. Sin embargo, a nivel social y normativo se ha evidenciado un proceder similar con el excombatiente, pues profundiza en dicotomías que se resumen a "lo bueno" y "lo malo" o quién puede ser víctima y quién victimario; esto castiga la narrativa en la medida en que se asocian calificativos con acciones indiscriminadamente. Al 
respecto, el Centro Nacional de Memoria Histórica (CNMH) y la University of British Columbia (UBC) (2013) argumentan:

Uno de los problemas reside en que al posicionarse como víctimas puede llevar a silenciar otras historias o aspectos de su experiencia, y en no abrir lugar para contar historias sobre cómo han sobrevivido, para sus historias de resiliencia, restablecimiento y resistencia [...] Este es uno de los silencios que aún no se ha revelado ampliamente y que llama a un proceso reflexivo y sensible de memoria y sanación colectiva. (pp. 19, 36)

Como señalan el CNMH y la UBC, leerse bajo determinada postura logra censurar o propiciar narraciones según sea el caso; por consiguiente, encontrar un punto neutro dentro de la narración fomenta la libertad dentro de ella. En este caso, el sentido de ser sobreviviente enlaza discusiones no solo disciplinares, sino cotidianas en el ciudadano de a pie que se encuentra inmerso en la experiencia del conflicto. No obstante, también se configura un reto disciplinar al reconocer posibilidades resilientes, corporales e identitarias, y cómo desde la psicología se articulan y construyen escenarios que favorecen la heterarquía, al contrario de la dicotomía que convoca la noción del ser excombatiente.

En la historia de Colombia el conflicto armado configura uno de los sucesos con mayor impacto sobre la población, pues en medio de la confrontación entre grupos armados ilegales y fuerzas militares se ha presentado continuamente la vulneración de los derechos humanos y se ha fracturado el Derecho Internacional Humanitario (DIH), lo cual ha dejado huellas importantes en términos económicos, políticos y sociales, pero también psicológicos (Tamayo, 2015).

Esta última cuestión evoca la imperiosa necesidad de abordar el conflicto armado desde una de las muchas caras de la guerra; la pluralidad de actores trae consigo la versatilidad de voces — puesto que no todos hemos vivido el conflicto en la misma medida- A este escenario se suman las conversaciones y diálogos de paz, firmados en el año 2016 por el gobierno de Juan Manuel Santos y por Rodrigo Londoño en representación del grupo Farc-EP (Gómez, 2017). 
Bajo esta lógica, los acuerdos de paz incorporan posibilidad de cambio y metamorfosis para toda la población, teniendo en cuenta que cierran la brecha visibilizada en la Ley de Víctimas y Restitución de Tierras o Ley 1448 de 2011 (Congreso de Colombia, 2011). Los parámetros de atención social y psicológica en medio del conflicto armado no contemplaban la población excombatiente; no obstante, este aspecto es acogido en el Acuerdo Final (Gobierno de Colombia y Farc-Ep, 2016), donde se reconoce la variedad de víctimas directas e indirectas, incluso los actores armados como los integrantes de las Farc-EP y los miembros de la Fuerza Pública. La invitación queda abierta a observar el sentido humano de los excombatientes que, si bien no justifica las acciones llevadas a cabo en el marco del conflicto armado, tampoco hace de ellas razones para el silencio y la obviedad de sus relatos.

\section{EMERGENCIA DE LA RESILIENCIA EN LA GUERRA}

Los estudios que vinculan al excombatiente desde su dimensión psicológica y humana son mínimos. Debido a esto, el ejercicio académico en contextos bélicos se hace complejo por su crudeza, ya que "la verdad" es un constructo de difícil acceso. En esta medida, las investigaciones y los procesos que se adelantan en este campo son un acercamiento a las diferentes versiones y sus actores como primera fuente.

La resiliencia es un concepto que ha estado vinculado a contextos bélicos de manera estrecha, ya que, como señala Cyrulnik (2005), cada guerra se moldea de una forma particular, similar a una personalidad, y en ese mismo sentido moldea la personalidad de aquellos a quienes toca. Al respecto, Cyrulnik ha adelantado diversos documentos en el campo de la resiliencia con nińos y adolescentes, en los cuales refleja que tras una situación de trauma — como una guerra—, el sujeto se ve moldeado y cambiado por él mismo, con lo que desarrolla el recurso resiliente. Viktor Frankl es un caso que personifica esta idea, pues a partir de su paso en los campos de concentración surge en él un sentido "real" de humanidad, y más adelante lo consolida como una filosofía de vida, siendo en la adversidad donde logra una comprensión profunda de sí mismo (Frankl, 1991). 
De manera concreta, en los estudios que se han adelantado sobre la resiliencia en contextos de guerra, se han usado diferentes estrategias y modelos. Por ejemplo, Guzmán et al. (2017) apuestan por la creación y aplicación del videojuego, donde se utilizan elementos creativos y actuales para acceder a poblaciones como los niños y los adolescentes. Como señala Acosta (2018), tras un análisis exhaustivo de la resiliencia en el conflicto armado colombiano, son muchas las estrategias y herramientas de las cuales hacen uso los investigadores para favorecer dicho recurso; en efecto, un aspecto que resalta la autora es la flexibilidad en la intervención comunitaria.

Por otro lado, las Fuerzas Militares del Estado también han estado ligadas a estudios relativos a la resiliencia y el factor psicológico. Particularmente, Torres (2012), en su investigación, utiliza el calificativo de "héroes" para los miembros de las Fuerzas Armadas, e invita a pensar y reflexionar sobre la representación social o lo que implica vestir un uniforme militar. En contraste, los estudios que abarcan afectaciones en excombatientes son escasos, aspecto que invita a reflexionar sobre la divulgación académica y las políticas públicas que existen frente a la población excombatiente.

Al respecto, Aristizábal, Howe y Palacio (2009) han sumado esfuerzos por denotar que la población combatiente y excombatiente no es ajena al sufrimiento que encara la guerra. En su estudio reflejan que bajo esta figura también se desarrollan afectaciones psicológicas y traumas, que se configuran de manera similar en militantes y víctimas, lo que ubica las formas de poder como una característica particular del suceso traumático.

Por último, en el marco del conflicto armado, el enfoque de género añade otros padecimientos como los delitos de índole sexual y el impacto social que llevan a cuestas las mujeres al ser quienes en muchos casos lideran la dinámica del hogar por su papel de cuidadoras en medio del conflicto, lo cual se resume en una capacidad de afrontamiento y resurgimiento (Albarracín y Contreras, 2017).

En esta misma línea, un estudio que retroalimenta de manera particular el papel de la mujer dentro del conflicto con el rol de combatiente y excombatiente es la tesis de López (2009) Las mujeres imaginadas de la guerra. Narraciones de excombatientes 
paramilitares sobre las mujeres y el conflicto armado. En este trabajo, da cuenta de la multiplicidad de significados que puede encarar y representar la mujer desde su posición como combatiente en la guerra. Ciertamente, la mujer no representa en la guerra el mismo rol que el hombre ya sea como víctima o como victimario, puesto que carga a cuestas con otras asignaciones y funciones. Lo que más resalta de su tesis es el desarrollo de la postura "inflexible" y "dura" de la mujer para representar su fortaleza, así como el papel del cuerpo y el factor emocional en representación del placer sexual y el apoyo moral. A pesar de su data, este estudio trae a discusión elementos muy pertinentes que entran a jugar con los propuestos en esta investigación, pues ofrece elementos de la mujer militante en un primer plano, como la fortaleza, la sexualidad y la guerra, que pueden surgir en medio de la narrativa bajo la cual se comprende la resiliencia en torno a la corporalidad e identidad.

\section{Hermenéutica y CONSTRUCCIONISMO DE LA GENÉTICA SOCIAL}

A nivel teórico, se resalta la complementariedad y la línea de trabajo bajo la cual se inscribe el enfoque histórico-hermenéutico y el enfoque crítico social a partir del construccionismo como paradigma teórico de la psicología, ya que estos enfoques comparten una visión de proceso.

La intervención in situ a la cual invita el enfoque crítico es antecedida por la interpretación de la realidad o fenómeno en los cuales se ejecutan los procesos movilizadores. Por su parte, el enfoque histórico-hermenéutico está integrado por las visiones del contexto que integran la interpretación; por ende, la temporalidad es un elemento que se debe considerar de forma más profunda. Como bien explica Ramos (2018), articulando los postulados de Gadamer con los de su discípulo Koselleck, pasado, presente y futuro se encuentran en constante interacción ya que el ser humano busca comprender no solo los hechos que lo anteceden — pasado-, sino también su desarrollo próximo - futuro-, en las formas del lenguaje a través de las cuales se interpreta el ser humano. 
Justamente, genética social es el concepto propio para definir y denotar los rastros histórico-sociales presentes en las dinámicas de la cotidianidad a nivel colectivo e individual. La genética social establece que a pesar de que determinados hechos se ubiquen en una temporalidad lejana, con el trascurso de los años subsisten rastros de estos mismos que se heredan a través de las generaciones, de modo que existe una expresión fenotípica de hechos del pasado en la actualidad. Este fenómeno, si bien no ubica la historia como un círculo vicioso infinito, halla patrones que se reproducen regularmente a nivel social y personal, por ejemplo, los lapsos de violencia sociopolítica de Colombia.

En relación con esto, Ramos (2018) abarca otros referentes sobre la hermenéutica de Gadamer, como la relevancia de la historia y la experiencia en tanto elementos cruciales. Estos funcionan como ejes centrales para ubicar otros aspectos del análisis hermenéutico, como el prejuicio, la autoridad y tradición, la historia y la conciencia efectuales, observados finalmente en el producto de dicho análisis, también reconocido como metacomprensión narrativa.

A partir de lo anterior, el construccionismo social edifica dicha metacomprensión para narrar en otro sentido la experiencia vital. En esta corriente de pensamiento: "[...] el fundamento se pone en el discurso como vehículo a través del cual se articulan el yo y el mundo, y en la forma en que funciona tal discurso dentro de las relaciones sociales [...]" (López-Silva y Otaíza, 2015, p. 581).

Como representante de esta postura, Gergen (1996a) fundamenta el construccionismo social como una mirada que abarca las ciencias sociales y la psicología, desde la crítica social sujeta a la crítica literaria e ideológica. También, resalta el valor de encontrar una visión que da cuenta de las versiones humanas desde las relaciones sociales a partir de las cuales se construye el mundo.

Ahora bien, el construccionismo social, desde los postulados de Gergen, ofrece también la posibilidad de ser en el lenguaje en distintas formas y tiempos, y da paso incluso a formas autorreferenciales para el investigador, posibilitando escenarios heterárquicos y amplios en narrativas reflexivas, siendo esta reflexión la apertura a un 
proceso más profundo conceptualizado como el proceso autorreferencial: "Se podría traducir como referirse a sí mismo sobre el otro" (Rodríguez, 2008, p.85). Similar es la postura de Estupińán y González (2012), al referir que la autorreferencia implica un ejercicio activo en el vínculo relacional en donde se reconoce la otredad y lo que surge en torno al momento de interacción que se ha gestado como elemento que no es ajeno a la postura de los actores.

A lo largo de la investigación se elaboró una juiciosa conceptualización de los principales términos trabajados: resiliencia, corporalidad e identidad, narrativa y relato. En principio, la resiliencia se vinculó particularmente a las nociones ofrecidas por el autor Cyrulnik y su concepción de oxímoron adaptable a diferentes escenarios dentro de este mismo ejercicio, equiparable al Yin-Yang. Para precisar, Cyrulnik (2006) indica que:

[...] el oxímoron revela el contraste de aquel que, al recibir un gran golpe se adapta dividiéndose. La parte de la persona que ha recibido el golpe sufre y produce necrosis, mientras que otra parte mejor protegida, aún sana pero más secreta, reúne, con la energía de desesperación, todo lo que puede seguir dando un poco de felicidad y de sentido a la vida. (p. 21)

En segundo lugar, la corporalidad e identidad son el elemento físico de lo mental. El cuerpo es evidencia de la experiencia vital, reconocido y comprendido por el mismo sujeto dentro de su esquema identitario y formula una correlación directa con aquello que es físico y psicológico. Por consiguiente, "el proceso de construcción de la identidad es inseparable de la materialización tanto en el cuerpo como en el discurso que lo reproduce" (Murillo, 2018, p. 29).

Finalmente, la construcción conceptual vinculó la narrativa y el relato partiendo del lenguaje como medio principal para llevar a cabo el proceso investigativo. En este sentido, la narrativa constituye la forma de expresión y representación de la experiencia vital, al igual que la realidad de los actores del contexto investigativo/ interventivo: "En un mundo construido y constituido por palabras existe una relación 
entre la vida y las narrativas, es decir que damos sentido narrativo a nuestras vidas y asimismo, damos vida a nuestras narrativas” (Domínguez y Herrera, 2013, párr. 5).

\section{Diseño METODOLÓGICO}

\section{Marco referencial}

La propuesta metodológica más pertinente para la investigación es de línea cualitativa y contempla, a su vez, la perspectiva lógica de la cibernética de segundo orden, postura elocuente con la premisa de la autorreferencia. La cibernética de segundo orden es comprendida desde la circularidad, teniendo en cuenta que en medio de la interacción quien hace las veces de investigador es mediado, "tocado" y visibilizado por otro (Von Foerster, 2012). Por otro lado, la investigación-intervención comporta un proceso de contextualización del fenómeno de estudio desde una postura relacional, reflexiva y contextual; de ahí que los procesos de reflexividad impliquen un sentido ético en el desarrollo de este tipo de escenarios, donde las voces son traídas de manera heterárquica y no jerárquica. En este proceso se incluye en todo momento al observador dentro de las observaciones, para así poder hacer investigaciones de segundo orden desde una apuesta generativa, recíproca y contextual (Estupiñán, 2003).

A nivel metodológico son también reflejados algunos de los postulados históricohermenéuticos y construccionistas. A nivel histórico-hermenéutico se habla sobre el horizonte, que da apertura a dos momentos: en primer lugar, que el participante tenga una comprensión de sí mismo como resultado de su conciencia histórica y, en segundo lugar, la posibilidad de favorecer la narrativa resiliente en la realización de horizontes próximos en su experiencia de vida. Todo esto está sujeto a que, si bien no puede sentirse de nuevo un evento pasado desde la experiencia física, y su significación no constituye una categoría universal, sí puede interpretarse y reinterpretarse desde diferentes puntos históricos.

En efecto, cada fracción de momento en estas temporalidades supone una posibilidad diferente de interpretación teniendo en cuenta el constante cambio del ser humano, 
el horizonte y la distancia. Como resultado, desde los postulados de Gadamer, el proceso hermenéutico concluye con la conciencia sobre el proceso histórico mismo, en la medida en que se comprende el fenómeno y el investigador desde su historicidad.

La posibilidad resulta de la interpretación en el proceso investigativo, lo cual favorece los escenarios de movilización bajo las formas de narrativa resilientes del actor. Gergen (1996b), como expresión de lo que se puede comprender como horizonte desde el enfoque histórico hermenéutico, describe tres formas básicas de narrativas: estables, progresivas y regresivas. La narrativa estable es aquella que no denota cambios abruptos en el discurso; la narrativa progresiva se identifica por una posibilidad de crecimiento discursivo de mejora y potencialidad, a diferencia de la narrativa regresiva, que sitúa una forma decreciente, cuyos aspectos no mantienen ni posibilitan la mejora en la escena relacional y discursiva, sino que por el contrario ubican al narrador desde una postura de deterioro (figura 4.1).

Figura 4.1. Interpretación visual de Gergen sobre las formas narrativas y el proceso de macros y micro narrativas.

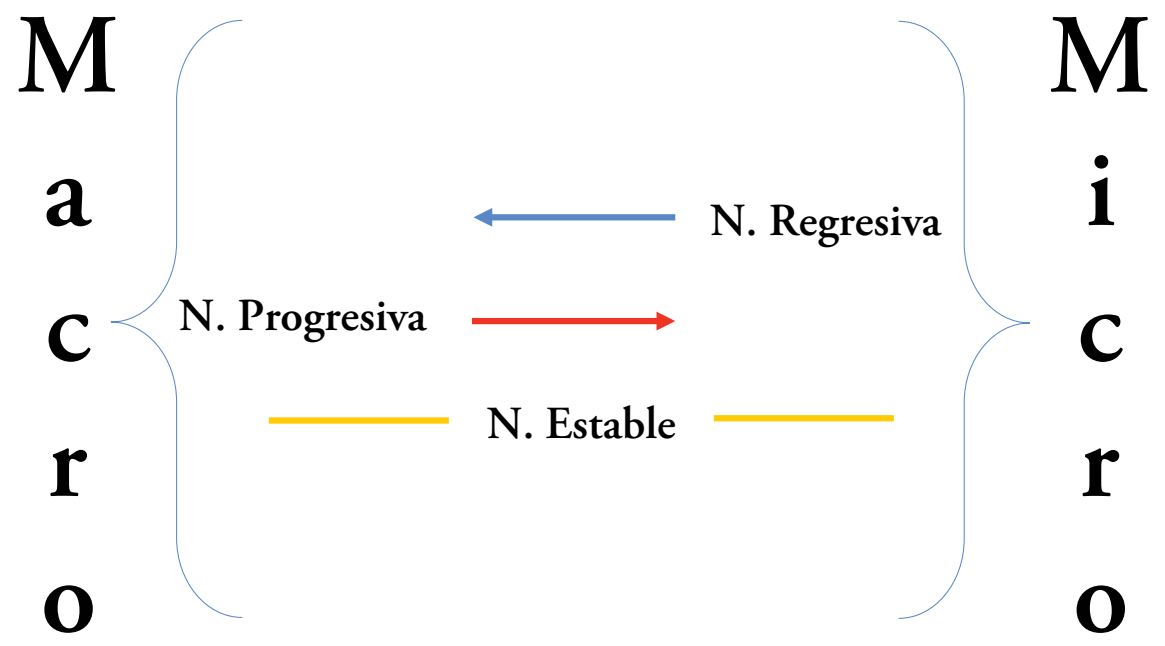

Fuente: elaboración propia, a partir de Gergen (1996b). 
En la visión de Gergen (1996b) también se incluyen algunas figuras literarias que surgen como resultado de la combinación de estos momentos, como por ejemplo la representación de la "tragedia", donde el punto (A) es la narración progresiva y el punto (в), la narración regresiva en un lapso corto. Por el contrario, en la "comedianovela”, el punto (A) lo ocupa la narración regresiva y el punto (B) pertenece a la narración progresiva, enmarcadas en micro o macro narraciones (figura 4.2).

Figura 4.2. Interpretación visual de Gergen sobre las lógicas de la forma narrativa a través de las figuras literarias de la tragedia y la comedia-novela.

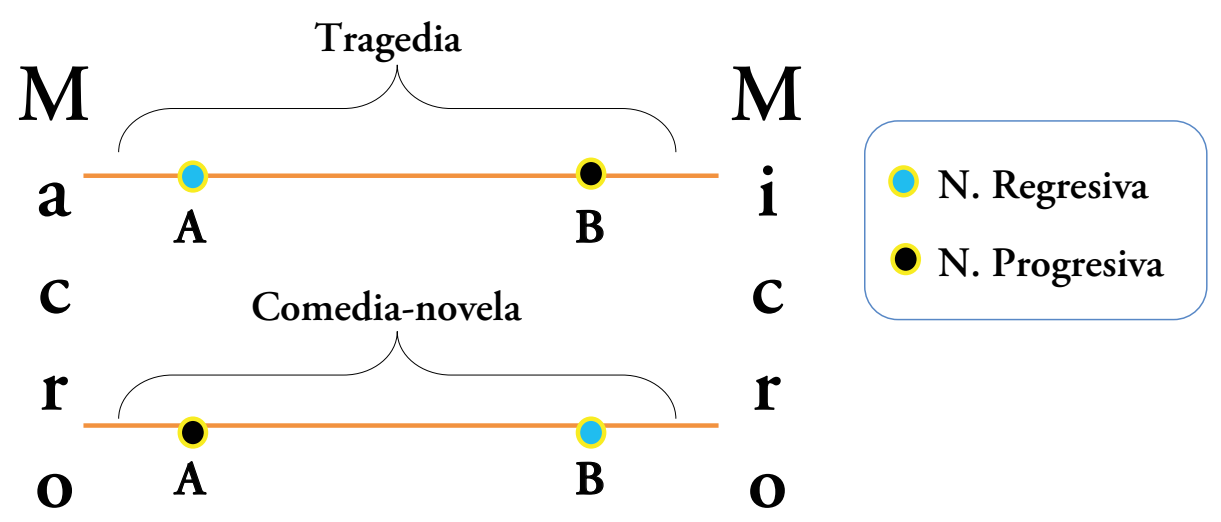

Fuente: elaboración propia, a partir de Gergen (1996b).

Lo anterior es pertinente teniendo en cuenta el lenguaje como expresión del mundo. De este modo se posibilitan las formas narrativas a través de la historicidad, con el fin de ampliar la comprensión de la resiliencia en el excombatiente y como herramienta fundamental para el hallazgo y fortalecimiento de narrativas progresivas que dan cuenta de la trasformación positiva y la resiliencia en el participante. Esta posibilidad también da paso al investigador, quien, desde su rol como sujeto, con la mirada del prejuicio y de su propia historicidad, puede contemplar dentro de sí la posibilidad transformadora, lo que da lugar a aspectos autorreferenciales. 


\section{Categorías iniciales}

Las categorías iniciales emergen a partir de la comprensión teórica y epistemológica, ya que abarcan diferentes elementos y postulados de la recolección de información durante dichos momentos. Ahora bien, la categoría denominada "distancias resilientes" (figura 4.3) hace referencia principalmente a la distancia histórica que representan cronológicamente el participante y el investigador. El descubrimiento de esta distancia implica que se integren narrativas que posibiliten la resiliencia a partir de la figura del oxímoron, de modo que se genere como resultado un entendimiento sobre sí mismo y dicha historicidad.

Figura 4.3. Codificación y construcción de la categoría inicial "distancias resilientes".

\section{Distancias resilientes}

\section{DR}

\section{Distancia}

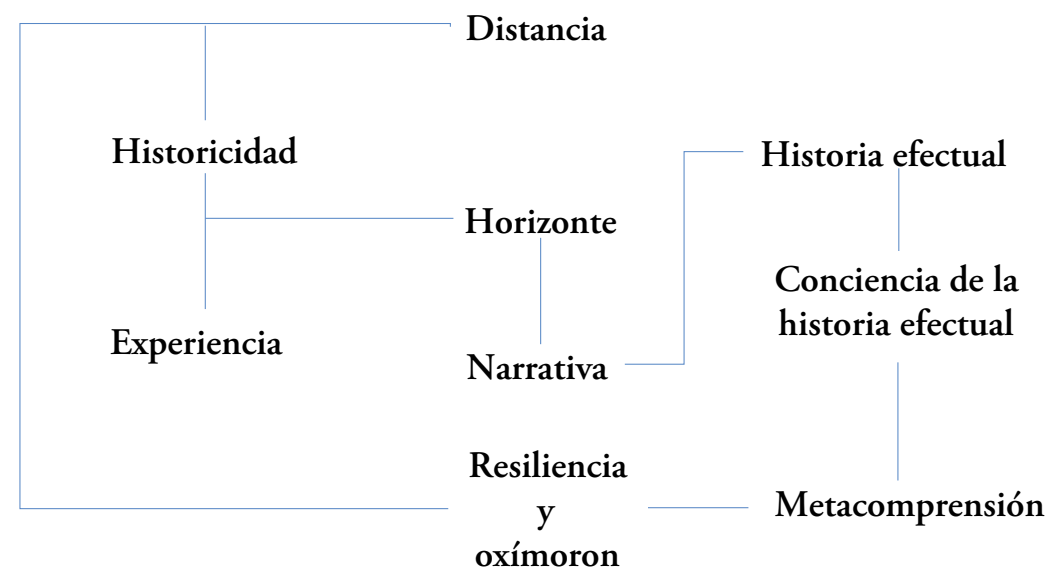

Fuente: elaboración propia. 
Por otro lado, "la épica herida de guerra" (figura 4.4) implica la lectura de aquellas realidades relacionadas directamente con el conflicto y la guerra, inscritos a nivel corporal e identitario desde los postulados narrativos ofrecidos por Gergen, a través de la tragedia y la comedia-novela; tiene como objetivo la visualización del oxímoron dentro de dichas realidades.

Figura 4.4. Codificación y construcción de la categoría inicial "la épica herida de guerra".

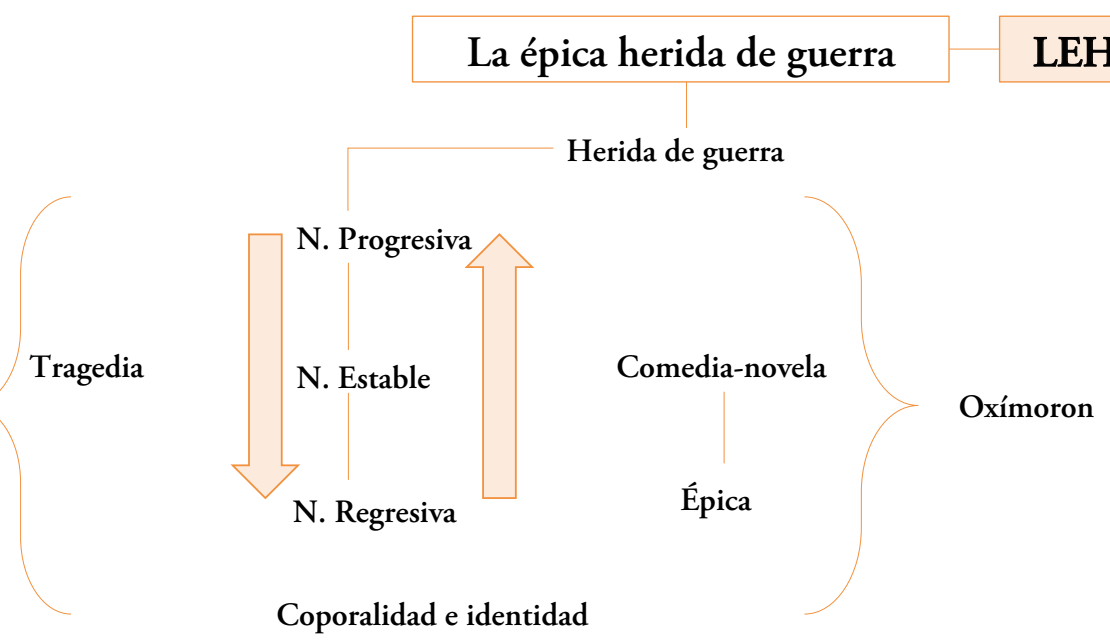

Fuente: elaboración propia.

Finalmente, la categoría denominada "conservación ideológica" (figura 4.5) hace referencia a las dinámicas que tuvieron lugar dentro de los escenarios conversacionales en los cuales se desarrolló la investigación. El punto de partida fue la distancia e historicidad desde la cual se leen e interpretan narrativas establecidas que dan cuenta de tradiciones y posiciones de autoridad reproducidas en la cotidianidad, y reconocidas a partir de un ejercicio reflexivo de metacomprensión y autorreferencia. Igualmente, en medio de esta categoría se da por sentado la dinámica heterárquica desde la cual se ha intentado llevar a cabo el ejercicio investigativo. 
Figura 4.5. Codificación y construcción de la categoría inicial "conservación ideológica”.

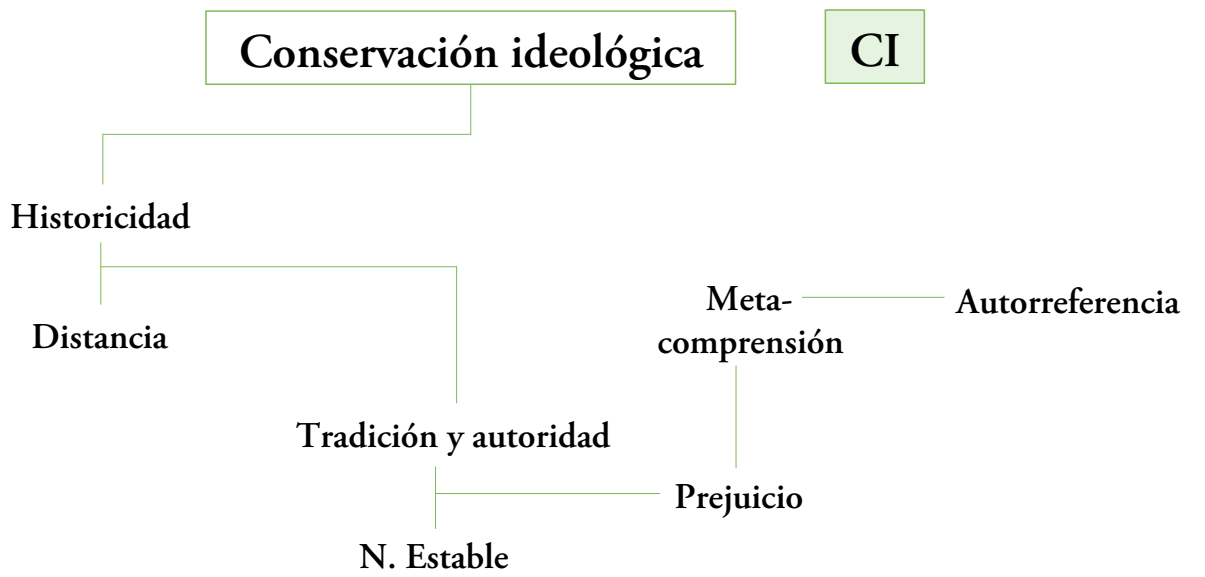

Fuente: elaboración propia.

\section{Desarrollo metodológico}

Para el desarrollo metodológico se utilizó la modelación teórica, con el fin de retroalimentar y mejorar cada escenario llevado a cabo. Dichos escenarios hacen referencia a los encuentros que se llevaron a cabo dentro del ejercicio investigativo, denominados escenarios conversacionales o escenarios sensoriales, según haya sido el caso. En cuanto a esta etapa del proceso es relevante mencionar que dicha modelación posibilitó planear y desarrollar los escenarios a partir de la experiencia de los anteriores, llevando un hilo conductor en todo el proceso. En este sentido, se organizaron cuatro escenarios o encuentros; en este caso, el primer encuentro se conceptualizó como un protocolo de escenario, pues allí se acordó la participación del excombatiente y se conversó brevemente sobre el ejercicio, aspecto generó inquietudes, posibilidades y pautas sobre la intervención.

Asimismo, las estrategias de los escenarios conversacionales se llevaron a cabo desde los recursos narrativos y sensoriales, a partir de los avances del Centro Nacional 
de Memoria Histórica (CNHM) y Fundación prolongar (2017): "Fue sorprendente constatar la manera como el cuerpo participa de los relatos, complementa, amplia, corrobora o enriquece la narración" (p. 11). Igualmente, el mapeo corporal en 3D resaltó la narrativa de la corporalidad gracias a elementos que evocaron el cuerpo dentro del relato con el uso de la plastilina para moldear el cuerpo. Respecto a esto, Murillo (2018) específica que, mediante las representaciones visuales — p. ej. gráficos y dibujos-, se pueden dejar en evidencia aquellas experiencias que han quedado marcadas no solo en la memoria; por esto, son una herramienta a priori para que se dote de una voz al cuerpo y se de paso a su relato.

Por otro lado, el tratamiento de las narrativas construidas en los escenarios conversacionales se analizó con elementos pragmáticos, semánticos y semióticos del discurso; este último tuvo lugar en el reconocimiento social y cultural, así como en las estructuras que conservaron su permanencia en medio de la interacción narrativa. La semiótica, como análisis de lo conversado, trajo a discusión la conexión de los sentidos y significados que tomaron lugar en los escenarios y las formas operacionales como productos de la praxis humana (Estupiñán, 2012; Hernández, 2008). Durante el análisis de los escenarios conversacionales se creó una nomenclatura sobre los diálogos que se generaron en los escenarios conversacionales y sensitivos, con el fin de ubicar de forma sencilla aquellas narrativas que ejemplificaban cada categoría (Figura 4.6).

Figura 4.6. Nomenclatura en el análisis y ejemplificación de datos.

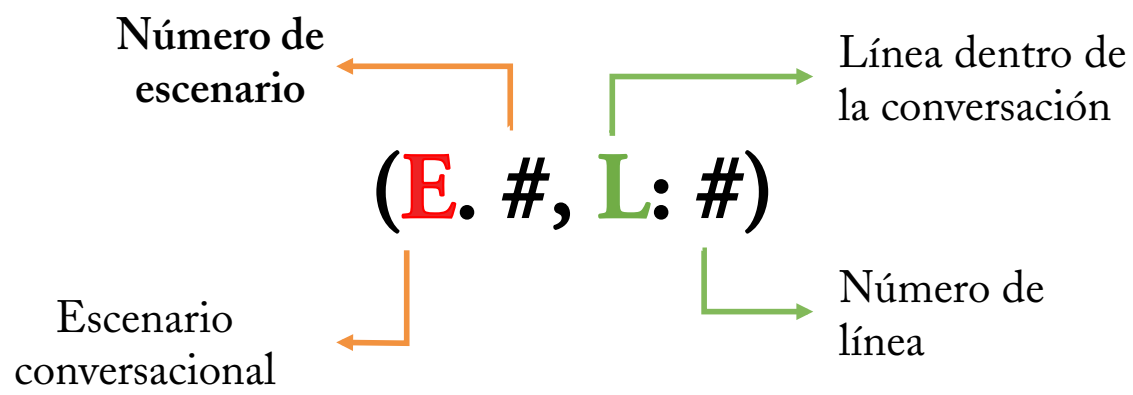

Fuente: elaboración propia. 


\section{Participante/actor}

La presente investigación cuenta con la participación de una mujer excombatiente perteneciente al grupo de las Farc-EP, quien para efectos del mismo proceso investigativo será reconocida con el alias que usó en sus años de militancia "Juana Alape", a lo cual ella accedió de manera voluntaria con el fin de proteger su seguridad.

\section{Resultados}

Los resultados se obtuvieron a partir del análisis planteado anteriormente y la categorización de relatos, ilustrados en fragmentos narrativos surgidos en medio del proceso conversacional. Fueron agrupados en las categorías iniciales y conceptualizados a partir de los significados más explícitos, que denotaron el sentido resiliente de la corporalidad e identidad en el marco del conflicto armado.

En cuanto a la categoría inicial "distancias resilientes", surgieron relatos desde la emocionalidad y la reflexión, aspectos que al parecer orientan la vida de la participante y que son el resultado de las vivencias y experiencias surgidas tras su paso por el grupo armado. Respecto a esto, la camaradería, la salud mental y la existencia misma son contempladas en este apartado.

JA: La moral, hablando, siendo conscientes de las cosas. (E. 3, L: 179)

IN: ¿Entre todos? (E. 3, L: 180)

JA: Sí, claro. Nosotros, la disciplina de nosotros, reuniones por la mañana, por la tarde. Bueno, nos toca esperar esto, esto y esto, alistemos, esto es lo que va a pasar y lo que pase fuera de eso ahí lo llevamos y ya. (E. 3, L: 181)

Ahora bien, al igual que se halla una construcción narrativa respecto a la moral, se da cuenta del sentido del equilibrio como un relato relacionado con la salud mental y el bienestar psicológico como factor relevante para sostenerse en la guerra y en la cotidianidad: 
JA: Se siente, si queda en cero, pero no sé si sea un equilibrio quedar en ceros, no sé. [silencio corto] Para mí el cero nunca ha sido la mitad, el cero es el cero, no tiene nada y el equilibrio no creo que sea eso, yo creo que el equilibrio es, por ejemplo: tú estás en una cicla y tú nunca estás así, tienes que pedalear a este lado y pedalear al otro [...]. (E. 4, L: 484)

Asimismo, la vida y la muerte se reflejan mediante la metáfora de renacer constantemente. El equilibrio mencionado anteriormente puede incluso ser un detonante en esta ecuación como lo refleja el siguiente relato:

JA: Sí, yo creo que, y soy consciente y totalmente creyente en que nosotros tenemos que sabernos morir y saber volver a vivir, no solo por situaciones, sino por momentos, por personas, por todo, [...] tenemos miedo a empezar de cero siempre. (E. 4, L: 269)

La categoría "épica herida de guerra" arrojó narraciones que se instauraron en el cuerpo como marcas físicas, propias de las dinámicas del conflicto y la vida en la selva. Al respecto surgieron significados y apropiaciones identitarias con la militancia:

JA: [...] Yo, cuando hablo de militar, es ¿por qué?, porque es que cuando, no solo militares sino una militancia, ¿sí?, ¿por qué la diferencia de los civiles? Porque un civil nunca va a tener un compromiso social, nunca va a tener un compromiso del todo con alguien con el que está a su alrededor. ¿Sí? De las, pues, un compromiso de vida hablo yo, de dar la vida.

Igualmente, surgieron construcciones hacia el reconocimiento de las fuerzas armadas del Estado como "héroes". Esta categoría también ofreció como resultado el reconocimiento del combate y el fusil como un momento de crisis y afectación psicológica por la densidad de las situaciones que en medio de este se generaban. El fusil también cumplía una labor importante en dos sentidos: en primer lugar, como la herramienta bélica con la cual se hería al otro y, en segundo lugar, como la protección que representaba como aliado protector. Por último, los hallazgos establecieron la práctica del tatuaje como una nueva herida de guerra, pues la 
participante tapaba sus heridas de guerra con estos, con lo cual los dotaba de otros sentidos y significados.

La conservación ideológica se observó a partir del movimiento interventivo conceptualizado como el conocimiento y liberación del prejuicio, donde se reconoce al interlocutor en medio de la narración dentro y fuera de su rol, y se acepta fuera de la estigmatización. Por otro lado, la participante estableció su posición frente a la realidad nacional, aspecto que estuvo marcado por los acontecimientos que se han presentado en relación con los acuerdos de paz, como refleja el siguiente fragmento:

JA: Sí claro, creí en el proceso de paz, sabía que el pueblo iba a funcionar de esta forma, sabíamos que el Gobierno iba a funcionar de esta forma, sé que el pueblo es ignorante, que el pueblo es cruel, pero hay algo tan en el fondo que todavía me tiene acá, que es que en algún momento esa hipocresía, esas falsas necesidades van a reventar [...]. (E. 4, L: 498)

Anexo a estos resultados se hallaron otros que no estaban contemplados dentro de las categorías iniciales, por tal razón se ubicaron como categorías emergentes, puesto que surgieron cuando la participante amplió otras dimensiones de su vida. A así, el vínculo familiar en la guerra fue referido por la participante en dos direcciones: en primer lugar, su núcleo familiar, y la posición y participación de ellos frente a las decisiones de Juana y, en segundo lugar, la relación y el vínculo construido con otros guerrilleros con quienes ella compartió durante su militancia.

Otro relato emergente surgió en relación con la filosofía de vida de Juana: "vivir al día”, ya que en su experiencia como combatiente no había certezas sobre los días venideros. Por último, la transición y el proceso interventivo se valoró como un resultado emergente teniendo en cuenta los movimientos heterárquicos y la apertura autorreferencial. Esta dinámica facilitó la construcción de metáforas que dieron cuenta de un sentido profundo y reflexivo de la experiencia vital. En un principio, los escenarios se regían por narrativas concretas y puntuales; sin embargo, cuando el investigador amplió la información de sí mismo frente al participante, 
este replicó el movimiento narrativamente a tal punto de generar un contexto investigativo-interventivo totalmente abierto.

\section{Discusión}

Inicialmente, la investigación se formuló en torno a la construcción narrativa de la resiliencia en relación con la corporalidad e identidad en un excombatiente de las Farc-EP. El problema se desarrolló a través de la apuesta epistemológica por establecer la complementariedad entre posturas histórico-hermenéuticas y críticosociales, en razón de ubicar los antecedentes y la posibilidad de trasformación en el ejercicio investigativo. Como resultado, los postulados referidos por Gadamer y Gergen fueron replicados de manera constante, e incluso llegaron a establecer conexiones con otros autores y cobraron sentido en el desarrollo de los escenarios conversacionales.

Las formas narrativas de Gergen (1996b) cumplieron un rol relevante dentro del desarrollo de los escenarios conversacionales, por cuanto guiaron el proceso interventivo y los movimientos que trasformaban el contexto de investigaciónintervención; junto con los fundamentos histórico-hermenéuticos de Gadamer, donde la distancia entre las partes (investigador y participante) se estableció un suelo fértil para la evocación de narrativas. La distancia posibilitó posturas autorreferenciales y heterorreferenciales en el escenario, donde se construyeron narrativas que oscilaban entre las diversas formas referidas por Gergen.

En cuanto a esto, las narrativas no siguieron la configuración referida por el autor frente a la tragedia y la comedia-novela. En cambio, establecieron como punto de partida la narrativa estable desde lecturas identitarias que contemplaban la militancia como un continuo de la historicidad y el horizonte en la autonarración desde la militancia, aun después de la dejación de armas y la vinculación al proceso de paz y el desarme de las Farc-EP. 
En medio de esta estabilidad mediaban puntos regresivos y progresivos en el relato que la ubicaban en un fuerte proceso identitario militante; sin embargo, los puntos de transición frente a qué narrativa se encontraba en qué punto del horizonte histórico fueron difusos, pues las narrativas regresivas también tenían matices estables. Esto se replicaba en la narrativa progresiva desde la normalización de hechos, situaciones y experiencias relacionadas a la posición de vivir al día.

Esta dificultad para establecer los puntos de corte definitorios entre una y otra narrativa fue comprendida desde el principio de la complejidad conocido como "la borrosidad", puesto que el principio opera en el ejercicio de los puntos medios en las lecturas de la realidad y el comportamiento, y reconoce otras posturas fuera de las posiciones dicotómicas, lo que argumenta la posibilidad de graduantes que no obedecen a los puntos extremos de la polaridad (De la Villa, 2017).

Esto sugiere que, en medio de las narrativas estables, regresivas y progresivas, existen otras formas más allá de la tragedia y la comedia-novela; por consiguiente, queda el interrogante sobre qué otras combinaciones y formas de expresión narrativa se están obviando al solo establecer la tragedia y la comedia-novela. Como se evidencia, existe una diversidad expresiva y experiencial dentro del acto narrativo, que plantea la posibilidad de otros graduantes narrativos y, en dado caso, otras formas narrativas fuera del progreso, el retroceso o la estabilidad. Adicionalmente, desde esta postura, existe la posibilidad de reflexionar frente al ímpetu de la identidad militante en el relato, así como la existencia de narrativas alternas a estas que sitúen a Juana fuera de la militancia, es decir, en otros planos de su vida.

\section{La resiliencia}

La resiliencia se observó en los escenarios conversacionales a través de la figura del oxímoron sugerida por Cyrulnik (2006). Este concepto refería posiciones contradictorias dentro de un mismo elemento, similar a "la maravilla del dolor". Con ocasión a dicho elemento, Juana reconstruyó narrativamente sus experiencias desde posturas generativas en medio de las vicisitudes, por ejemplo, el dolor frente al aprendizaje. 
El oxímoron también se hiló a partir de metáforas que daban cuenta de la conciencia de la historia efectual y la metacomprensión construida en el escenario conversacional, ya que surgían comprensiones sobre la experiencia vital y el aprendizaje obtenido en medio de dicha experiencia. Esto ubica a Juana, dentro de su horizonte, en una lógica generativa de su vida, pues resaltaron aquellos aspectos que fortalecieron su vida al ingresar a las Farc-EP.

En cuanto a esto, la metáfora expresó la posibilidad de renacer en medio de la muerte, semejante a los ciclos de la naturaleza y su extinción para reiniciar en otras formas de vida. Ahora bien, la muerte y la vida también fueron referidas cuando la tierra hablaba sobre los muertos y Juana retomaba el ritual de enterrar los muertos para que tomaran vida en medio de la tierra, pues el cuerpo dotaba de otros componentes el suelo, traducido a otras formas de vida.

La metáfora de vivir y morir fue el reflejo del oxímoron pues encarnó dos posturas incompatibles dentro de un mismo elemento traducidas en "sobrevivir". Esto trascendió las barreras de la finitud y permitió conceptualizar formas de vivir que escapan a la corporalidad y al cierre de ciclos, ya que funciona como proceso resiliente dentro de la capacidad de la participante para abandonar un ciclo vital, nacer y crecer en otro. Dicha capacidad también se replicó en la corporalidad e identidad y las heridas de guerra, en razón de que estas últimas quedaron marcadas en el cuerpo como huellas del conflicto armado; son mapas vivientes que hablan por sí solos frente a su sentir en la guerra.

Las heridas de guerra no solo están marcadas en el cuerpo a través de lo visible — de la cicatriz-, sino que también se instauraron en la memoria. Por esto, no se habla de solo un "cuerpo" desde las nociones biológicas, sino de la corporalidad como la unión de lo biológico y lo psicológico, en donde el cuerpo narra las heridas que desde el dolor y el llanto recuerda. En torno a esto, se complejiza el relato ya que la narrativa expande la noción de herida de guerra hacia otros aspectos que oscilan entre lo visible e invisible, lo que deja marcas permanentes en el cuerpo y lo que no, aquellas que son el resultado de enfrentamientos, caminatas y prácticas de lo que 
implica ser guerrillero como parte del sentir identitario. Sin embargo, todas hablan desde un mismo terreno: el de la memoria.

Por consiguiente, la noción metafórica de la corporalidad como dispositivo narrativo resiliente es la clave en esta investigación. La herida de guerra es el puente entre la corporalidad y la memoria en la emergencia narrativa de la resiliencia, como sucede por ejemplo al dotar de nuevos significados las heridas por medio del tatuaje, frente a la resiliencia del cuerpo.

\section{Analogías del sentir y la experiencia vital}

La analogía se evocó como terreno reflexivo de la experiencia vital y el sentir de la participante para ejemplificar la profundidad de la narrativa con el fin de que fuese comprensible la vivencia y la caracterización resiliente. En medio de esto, el recurso natural potencializó metacomprensiones sobre elementos cruciales en la experiencia vital de la participante frente al proceso resiliente, lo que hizo complejos la experiencia y el relato de la participante.

Inicialmente, los recursos son el ejemplo de la complementariedad desde diferentes dimensiones de un mismo componente, así como la posibilidad de reconocer las diferentes facetas de la realidad y la vida propia en mediación del equilibrio sostenido en la lógica del no-equilibrio, como refirió Juana en la analogía del montar bicicleta pedaleando de un lado y del otro. Esta conceptualización es leída bajo las estructuras disipativas en razón de que esta lógica comprende la dinámica entre el caos y el orden como elementos que interactúan de manera constante en la posición de mediar entre sus posiciones para dotar de significado el sentido de la vida (Maldonado y Gómez, 2011).

En este proceso dinámico se comprende no solo el plano mental, sino el plano de la realidad en comunicación con la individualidad. Ahora bien, esto sugiere una reorganización de las estructuras que median entre los procesos que reciben y entregan en el juego relacional, entendido como neoequilibrio: "[...] El resultado de sistemas dinámicos que aprovechan al máximo las oportunidades y bienes a su 
alrededor que los transforman y, en esa misma medida, generan tanta entropía como beneficio obtienen del medio ambiente" (Maldonado y Gómez, 2011, p. 20).

\section{Las metáforas en el contexto investigativo-interventivo}

Finalmente, otro elemento importante es el sentido de la resiliencia en la narrativa de Juana, que surgió en torno a la construcción de metáforas. Esto, como se ha evidenciado, reconfiguró la noción relacional en el escenario y la narración desde un punto reflexivo sobre la resiliencia; en medio de este, ha situado la corporalidad e identidad como facilitadores. Así mismo, la metáfora desbordó la postura inicial sobre la corporalidad como posibilidad narrativa de otras formas resilientes, e integró al escenario otros puntos de vista que redefinieron el fenómeno en un sinfín de posibilidades narrativas para abordar la resiliencia.

En razón de esto, surgen elementos como la filosofía carpe diem ante la amenaza latente de perder la vida. El compromiso asumido desde la identidad militar configuró la forma como la que la vida es vivida y sentida. Esta lógica fue también comprendida desde posturas que parten de la incertidumbre sobre los acontecimientos venideros. Este principio es propio de la realidad, razón por la cual no se configura desde características individuales (Maldonado, 2013).

El principio de la incertidumbre se vinculó a los resultados obtenidos en dos sentidos. En primer lugar, como la génesis de la filosofía carpe diem, ya que surgió como respuesta a la no certeza de futuros cercanos y contar solo con las posibilidades del momento. en segundo lugar, también se ejemplificó con relación a la vulnerabilidad del contexto bélico puesto que las posibilidades se restringen a cortos momentos dentro de este contexto, como sugirió el significado que esconde el fusil como aliado en medio de la incertidumbre del combate.

Las metáforas favorecieron el proceso resiliente al evocar otras formas del relato emergente, por medio del cual el participante es autónomo en la interacción que instaura entre la narrativa y su propia experiencia, y alude a metacomprensiones complejas al hilar y evocar elementos novedosos dentro de la construcción narrativa. 
Igualmente, la metáfora fue el resultado del proceso de acoplo al contexto investigativointerventivo, pues como se observó, ubicar este punto en las narrativas sugiere un proceso meticuloso de movimientos autorreferenciales y heterorreferenciales que se hacen realidad en el fortalecimiento del vínculo relacional investigador-participante desde la heterarquía, la historicidad y la distancia.

\section{Conclusiones}

La investigación planteó dentro de su lógica la visibilización humana de los excombatientes ante conceptualizaciones duales que orientaron discusiones que ubicaron a la psicología en una orientación polarizada en el marco del conflicto armado. Sin embargo, a raíz de los acuerdos de paz, Colombia se encuentra en un proceso de transición frente a las muchas formas de comprender el conflicto armado y su impacto en los pobladores; de ahí que esta investigación tenga implicaciones científicas y pacifistas.

En primer lugar, el fenómeno de estudio sugiere un reto ante el quehacer de la psicología dentro de los contextos sociales e históricos, por las problemáticas que lo acontecen, así como las características que lo hacen sostenerse en el tiempo. En este sentido, el conflicto armado no solo configura una problemática de diversas dimensiones, sino que es la génesis y el resultado de situaciones que evidentemente no funcionan en la sociedad, y que no son visibilizadas con la relevancia suficiente en las discusiones académicas.

Por su parte, las poblaciones hacia las cuales se han guiado las estrategias psicosociales para reconstruir el tejido social han obviado a los combatientes y excombatientes por su papel activo como actores del conflicto. Frente a esto, los excombatientes también reflejan el impacto humano de la guerra en el plano de lo psicológico, ya que es un contexto en el que están inmersos y se configura incluso desde estructuras básicas de la sociedad como la familia.

El trabajo psicológico con la población excombatiente ofrece un suelo fértil para la construcción de paz desde la academia. En relación con esto, el presente estudio 
ubicó como fenómeno principal la construcción narrativa de la resiliencia desde la corporalidad e identidad, dispositivos narrativos para su comprensión. Al respecto, la construcción narrativa emergió a raíz de la herida de guerra como huella del conflicto en la individualidad del participante, lo que reconfiguró la idea de guerra desde otros espacios enmarcados en la memoria y materializó la resiliencia en la corporalidad del participante dibujando la posibilidad psicológica de cicatrizar e instaurar nuevos significados a esas marcas del conflicto.

Igualmente, la psicología es visible en las lógicas bélicas por el equilibrio como elemento de salud mental, que es visible en la narrativa resiliente como una forma de mantenerse sobre el horizonte, lo que le da énfasis en la relación de este con otros elementos que lo configuran dentro del antagonismo del no equilibrio y ubica a nivel experiencial la posición del oxímoron como figura resiliente.

Por otro lado, corporalidad e identidad evocan narrativamente aspectos identitarios de la militancia, donde la diferencia de grupos no es relevante pues sobrepasa el compromiso de vida asumido por el "militar", aspecto que marca una diferencia con el ciudadano de a pie. Esta configuración identitaria se ve inscrita en la corporalidad cuando en medio de las dinámicas de la guerra el cuerpo es herido y marcado, lo que se traduce también en el plano de lo psicológico en la manera como son recordadas y evocadas en la narrativa, y el impacto de hacerlo en medio del contexto investigativo-interventivo.

El recuerdo y el relato de la herida de guerra también hilan procesos emocionales de fondo que potencializan la reflexión frente a la experiencia vital e instauran metacomprensiones que se organizan en pro de la narrativa resiliente. Así mismo, abren paso a la temporalidad del participante y establecen puntos concretos en la historicidad, situación que evidencia el cambio de ciclos en la analogía de morir y renacer constantemente.

Por último, otro elemento que reconfigura la noción del fenómeno es el vínculo familiar, que es necesario en la medida en que estabiliza la narración del participante. Esto sugiere además una red de apoyo primaria frente a las adversidades que se 
comparten en medio de la guerra, acción que reafirma el vínculo hacía otros compañeros y la misma estructura -Farc-EP-. Desde este análisis, las Farc-EP, como estructura, ejemplifican la manera como se moldea el comportamiento y sentir social a través de las instituciones básicas de la sociedad, como la familia.

\section{REFERENCIAS}

Acosta, I. (2018). La resiliencia, una mirada hacia las víctimas del conflicto armado colombiano [tesis de especialización]. Universidad Santo Tomás, Bogotá, Colombia.

Albarracín, L. y Contreras, K. (2017). La fuerza de las mujeres: un estudio de las estrategias de resiliencia y la transformación en la ocupación humana de mujeres víctimas del conflicto armado en Colombia. Revista Ocupación Humana, 17(1), 25-38.

Aristizábal, E., Howe, K. y Palacio, J. (2009). Vulneración psicológica en víctimas y victimarios por efecto del conflicto armado en Magdalena, Atlántico, Cesar, Sucre y Bolívar. Revista de Psicología Universidad de Antioquía, 1(2), 7-26.

Aristizábal, E. (2015). Traumas asociados a violencia en excombatientes de grupos armados ilegales. Revista de Psicología Universidad de Antioquia, 7(1), 21-34.

Centro Nacional de Memoria Histórica y University of British Columbia. (2013). Recordar y narrar el conflicto. Herramientas para construir memoria histórica. Colombia: Imprenta Nacional de Colombia.

Centro Nacional de Memoria Histórica y Fundación Prolongar. (2017). Reconstruir y recordar desde la memoria corporal. Guía metodológica. Consultado en http://www.centrodememoriahistorica.gov.co/descargas/informes-accesibles/reconstruir-y-recordar_guiametodologica_accesible.pdf.

Congreso de Colombia. (2011, 10 de junio). Ley de Víctimas y Restitución de Tierras [Ley 1448 de 2011]. Diario Oficial n. ${ }^{\circ} 48096$. 
Cyrulnik, B. (2005). Los patitos feos. La resiliencia: una infancia infeliz no determina la vida (5. ${ }^{a}$ edición). España: Gedisa.

Cyrulnik, B. (2006). La maravilla del dolor: el sentido de la resiliencia. Buenos Aires: Granica.

De la Villa, M. (2017). Conceptos básicos del paradigma de la complejidad aplicados a la cuestión del método en psicología social. Summa Psicológica USTA, 14(1), 12-22.

Domínguez, E. y Herrera, J. (2013). La investigación narrativa en psicología: definición y funciones. Psicología desde el Caribe, 30(3). Consultado en http://rcientificas.uninorte. edu.co/index.php/psicologia/article/view/4455/6951\#n_2.

Estupiñán, J. (2003). Algunos principios orientadores en los procesos de investigación intervención y formación de terapeutas y consultores de familia. En J, Estupiñán, A, Hernández, D. Rodríguez, M. Polo, D. Garzón, M. Barragán, L. Rodríguez, ... H. Sandoval, Construcciones en psicología compleja. Bogotá: Universidad Santo Tomás.

Estupiñán, J. y González, O. (2012). Narrativa conversacional, relatos de vida y tramas humanas: hacia la comprensión de la emergencia del "self" en interacción en contextos ecológicos [avance de investigación]. Universidad Santo Tomás. Consultado en https://bit. ly/2u6ANgg.

Frankl, V. (1991). El hombre en busca de sentido. Barcelona: Editorial Herder.

Gergen, K. J. (1996a). La construcción social: emergencia y potencial. En M. Pakman. (ed.), Construcciones de la experiencia humana (vol. 1) (pp. 139-175). Barcelona: Gedisa.

Gergen, K. J. (1996b). Realidades y relaciones. Paidós Básica.

Gobierno de Colombia y Farc-EP. (2016, 24 de noviembre). Acuerdo Final para la terminación del conflicto y la construcción de una paz estable y duradera. Consultado en http:// www.altocomisionadoparalapaz.gov.co/procesos-y-conversaciones/Documentos $\% 20$ compartidos/24-11-2016NuevoAcuerdoFinal.pdf. 
Gómez, M. (2017, 18 de noviembre). Así está Colombia, un año después de las Farc. El tiempo. Consultado en https://www.eltiempo.com/politica/proceso-de-paz/efectosen-colombia-tras-un-ano-de-la-firma-del-acuerdo-de-paz-con-las-farc-152740.

Guzmán, A., Inchima, B., Ortiz, J., Mera, J., Encarnación, M., Gómez, R. y Mosquera, Y. (2017). Prototipo de videojuego para respaldar la resiliencia en menores de edad víctimas del conflicto armado en el Municipio de Caldono Cauca. Memorias de Congresos UTP. Consultado en https://revistas.utp.ac.pa/index.php/memoutp/article/view/1485.

Hernández, A. (2008). Vinculo, individuación y ecología humana. Hitos para una psicología clínica compleja [proyecto institucional de investigación]. Universidad Santo Tomás, Bogotá.

López, M. (2009). Las mujeres imaginadas de la guerra. Narraciones de excombatientes paramilitares sobre las mujeres y el conflicto armado [trabajo de pregrado]. Universidad Nacional de Colombia, Bogotá.

López-Silva, P. y Otaíza, M. (2015). Construccionismo social y el sujeto libre: sobre negaciones y consecuencias. Revista de Filosofía Aurora, 27(41), 569-593.

Maldonado, C. E. y Gómez, N. A. (2011). El mundo de las ciencias de la complejidad. Una investigación sobre qué son, su desarrollo y sus posibilidades. Consultado en https://www. ugr.es/ raipad/investigacion/excelencia/seminarioXV/2011_el_mundo_de_las_ciencias_de_la_complejidad.pdf.

Maldonado, E. (2013). Significado e impacto de las ciencias de la complejidad. Bogotá: Desde Abajo.

Murillo, K. (2018). Cuerpos haciendo memoria. Narrativas y corporalidad en la escuela superior de guerra en Bogotá [trabajo de pregrado]. Universidad Santo Tomás de Aquino, Bogotá, Colombia.

Ramos, F. (2018). Huella de Hans- George Gadamer en Reinhart Koselleck. Aportes a la historia conceptual. Historelo. Revista de historia regional y central, 10(19), 241-268. 
Tamayo, H. (2015, 12 de febrero). Conflicto armado en Colombia: factores, actores y efectos múltiples. El mundo. Consultado en https://www.elmundo.com/portal/noticias/derechos_humanos/conflicto_armado_en_colombia_factores_actores_y_efectos_multiples. php\#.Xo33wshKjDe.

Von Foerster, H. (2012). Ética y cibernética de segundo orden. En G, Nardone., y P, Watzlawick. (eds.), Terapia breve: filosofía y arte. Consultado en http://reader.digitalbooks.pro/book/preview/17691/html2301/-?1579396628827. 